\title{
Skyrmion states in thin confined polygonal nanostructures
}

\author{
Ryan Alexander Pepper, ${ }^{1,}$ a) Marijan Beg, ${ }^{1,2}$ David Cortés-Ortuño, ${ }^{1}$ Thomas Kluyver, ${ }^{1}$ Marc-Antonio Bisotti, ${ }^{1}$ \\ Rebecca Carey, ${ }^{1}$ Mark Vousden, ${ }^{1}$ Maximilian Albert, ${ }^{1}$ Weiwei Wang, ${ }^{3}$ Ondrej Hovorka, ${ }^{1}$ and Hans Fangohr ${ }^{1,2}$,b) \\ 1) Faculty of Engineering and the Environment, University of Southampton, Southampton SO17 1BJ, \\ United Kingdom \\ 2) European XFEL GmbH, Holzkoppel 4, 22869 Schenefeld, Germany \\ 3) Department of Physics, Ningbo University, Ningbo 315211, China
}

Recent studies have demonstrated that skyrmionic states can be the ground state in thin-film FeGe disk nanostructures in the absence of a stabilising applied magnetic field. In this work, we advance this understanding by investigating to what extent this stabilisation of skyrmionic structures through confinement exists in geometries that do not match the cylindrical symmetry of the skyrmion - such as as squares and triangles. Using simulation, we show that skyrmionic states can form the ground state for a range of system sizes in both triangular and square-shaped FeGe nanostructures of $10 \mathrm{~nm}$ thickness in the absence of an applied field. We further provide data to assist in the experimental verification of our prediction; to imitate an experiment where the system is saturated with a strong applied field before the field is removed, we compute the time evolution and show the final equilibrium configuration of magnetization fields, starting from a uniform alignment.

\section{INTRODUCTION}

Magnetic skyrmions have been an active research area in recent years after theoretical predictions of formation in materials with broken inversion symmetry, which host a Dzyaloshinskii-Moriya (DM) interaction.13 These predictions have been experimentally realised in a variety of materials, such as in the bulk metallic cubic B20 materials $\mathrm{FeGe}^{4}$ and $\mathrm{MnS}{ }^{5} \sqrt[8]{8}$, the insulating $\mathrm{Cu}_{2} \mathrm{OSeO}_{3}{ }^{910}$, and in thin film and multilayered systems!11 Driving this research, aside from the interest in the physics of such systems, are potential engineering applications to data storage and logic devices. The application to data storage in particular is important due to current challenges in existing technology. The magnetic recording trilemma ${ }^{14}$ is a well known problem with domain based storage, whereby the shrinking of current domain sizes competes with potential data loss from thermal fluctuations, requiring magnetically stiff materials and a correspondingly higher write field, which becomes difficult to achieve. Magnetic skyrmions, which can be of a much smaller size than the current domains, are a potential solution to this problem because the topology of the magnetization can provide a larger energy barrier to destruction. 15-17 Experiments have shown that skyrmion creation and deletion can be achieved through the injection of spin polarised currents, and that skyrmion manipulation can be achieved with low current densities relative to magnetic domain walls. ${ }^{8 \mid 18] 19}$ Storage device proposals include racetrack based storage, where the presence or absence of a skyrmion system can represent a bit.15|20 Other skyrmion device proposals which have been investigated include spin-transfer oscillators. $21 \mid 22$

Recent studies have shown nucleation of skyrmions

\footnotetext{
a)Electronic mail: ryan.pepper@soton.ac.uk

b) Electronic mail: hans.fangohr@xfel.eu
}

at room temperature in bulk systems and in interfacial systems, which brings the goal of creating devices much closer.23124 The physics and energetics of confined geometries differ significantly from those of large bulk systems. ${ }^{25}$ This is of particular concern for magnetic systems because topological protection is not afforded to skyrmions in finite-sized systems; skyrmions can be destroyed via variation of the magnetization field at the boundary, with a significantly lower energy barrier than other skyrmion destruction mechanisms. ${ }^{17}$ To this end, it is important to understand how the confined nature of the geometry can affect the energetics of the skyrmion states. In a previous study, FeGe nanodisks were studied through micromagnetic simulations, and it was found that skyrmion states could form the ground state in a narrow range of disk sizes, with no applied magnetic field ${ }^{27}$ This demonstrates a stabilisation of the skyrmion via the sample boundary. Another study focused on the effects of finite lateral dimension and thickness of a rectangular faced Cobalt nanowire, hosting an interfacial DMI $\stackrel{28}{20}$ Here, it was shown that skyrmions form in wires only for certain ratios of side length and above a certain size, and in such systems variation of the anisotropy and DMI strength can enhance or decrease skyrmion stability.

Recently, experimentalists have created FeGe nanodisks, and have observed skyrmion cluster and target states, 2,290 in line with theoretical predictions $27 / 31$. However, it is not obvious that these results can be extended to other geometries, as the boundary of the system has a significant effect on the magnetization. In this paper we advance the understanding of skyrmions in confined geometries, by studying polygonal films of FeGe and investigate the ground and metastable states of these systems for a range of sizes and applied fields. We choose regular polygonal films in order to study how the shape of the systems affects the equilibrium states which form, and how this changes the lowest energy magnetization configuration at each system size. 
(a)

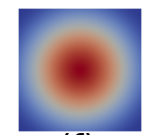

(f)

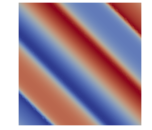

(b)

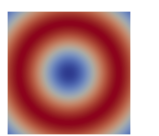

(g)

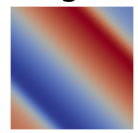

(c)

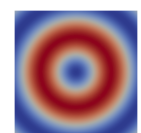

(h)

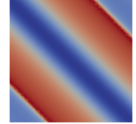

(d)

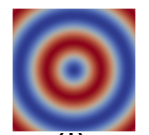

(i)

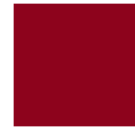

(e)

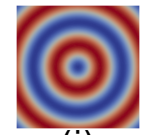

(j)

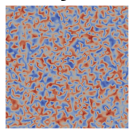

FIG. 1. Initial magnetization configurations from which each geometry is relaxed, shown here in a $140 \mathrm{~nm}$ side length film. The states are (a) incomplete skyrmion, (b) isolated skyrmion, (c) and (d) overcomplete skyrmions (e) target state (f), (g) and (h) helical states of different helical lengths (i) uniform state, and (j) random state.

\section{METHOD}

We study, through micromagnetic simulations, film systems of FeGe of thickness $10 \mathrm{~nm}$ using a fully threedimensional model. This model is chosen as it has been rigorously shown both theoretically and experimentally that in films of cubic helimagnets, chiral modulations occur along all three spatial dimensions, which reduces the skyrmion state energy in 3D systems of thickness lower than the helical length. ${ }^{32 \sqrt[35]{35}}$ The dynamics of the magnetization field $\mathbf{m}$ are modelled by the Landau-LifshitzGilbert (LLG) equation

$$
\frac{\partial \mathbf{m}}{\partial t}=\gamma_{0}^{*} \mathbf{m} \times \mathbf{H}_{\mathrm{eff}}+\alpha \mathbf{m} \times \frac{\partial \mathbf{m}}{\partial t} .
$$

Here, $\gamma_{0}^{*}=\gamma_{0}\left(1+\alpha^{2}\right)$ where $\gamma_{0}$ is the gyromagnetic ratio, and $\gamma_{0}<0$. The constant $\alpha$ is the Gilbert damping coefficient. The effective magnetic field is calculated as $\mathbf{H}_{\text {eff }}=-(\delta w / \delta \mathbf{m}) /\left(\mu_{0} M_{s}\right)$, where $w$ is the total energy density given as:

$$
w=w_{\text {Exchange }}+w_{\mathrm{DM}}+w_{\text {Zeeman }}+w_{\text {Demag }}
$$

The symmetric exchange energy density is $w_{\text {Exchange }}=$ $A(\nabla \mathbf{m})^{2}$ where $A$ is the magnetic exchange constant. The bulk Dzyaloshinskii-Moriya interaction (or antisymmetric exchange) in a material of crystallographic class $\mathrm{T}$ is given as $w_{\mathrm{DM}}=D \mathbf{m} \cdot(\nabla \times \mathbf{m})$ where $D$ is the DMI energy constant. The Zeeman energy is calculated from the applied field $\mathbf{H}$ as $w_{\text {Zeeman }}=-\mu_{0} M_{\mathrm{s}} \mathbf{m} \cdot \mathbf{H}$. The demagnetizing field is calculated using the Fredkin-Koehler hybrid FEM/BEM method $\stackrel{36}{26}$ For the simulations of FeGe, we use the parameters ${ }^{27} A=8.78 \times 10^{-12} \mathrm{~J} \mathrm{~m}^{-1}$, $D=1.58 \times 10^{-3} \mathrm{~J} \mathrm{~m}^{-2}, M_{s}=3.84 \times 10^{5} \mathrm{~A} \mathrm{~m}^{-1}$. The finite-element discretisation was set such that the distance between mesh nodes was no greater than $3 \mathrm{~nm}$, smaller than the relevant micromagnetic length scales for the given material, which has a helical length of $70 \mathrm{~nm}$ and exchange length $l_{\mathrm{ex}}=\sqrt{\frac{2 A}{\mu_{0} M_{s}^{2}}}=9.67 \mathrm{~nm}$

FeGe has an ordering temperature of $278.7 \mathrm{~K} .37 \mathrm{We}$ note that in large epitaxial thin-film samples of FeGe, studies showing skyrmion states have been performed within the temperature range of between $5 \mathrm{~K}$ and $275 \mathrm{~K}$ which show the skyrmion lattice state in the absence of an applied field 38 We further note that in the recent study by Zheng et. al. robust target states were observed in nanopillars of FeGe with Lorentz TEM measurements taken at $95 \mathrm{~K} \cdot 30$. With this in mind, we do not expect the effect of temperature fluctuations to be substantial, so long as experiments are done below the ordering temperature.

We compute the ground state phase diagram for two types of FeGe sample; square and triangular films of $10 \mathrm{~nm}$ thickness, through dynamic simulations. Dynamic simulations are used in order that all discovered states are physically realisable. We explore the energy landscape of structures by changing the applied magnetic field, which is varied between $0 \mathrm{mT}$ and $800 \mathrm{mT}$, and which is applied in the $z$ direction, into the plane of the system. In squares, we study films which have a side length between $40 \mathrm{~nm}$ and $180 \mathrm{~nm}$, and in triangles we use the broader range of side lengths between $40 \mathrm{~nm}$ and $220 \mathrm{~nm}$. Initially, the magnetization of each point in the phase space is set to each configuration of a set of initial states; the definition of these states is the same as those used in the study of Beg et al.27 (see Supplementary Material). The set of initial states, which includes uniform magnetization, skyrmionic state profiles, helical profiles, and a random magnetization state (which is repeated three times) are shown in Fig. 1. This systematic exploration is done in order to capture as many equilibrium states as possible for each simulated system. In order to construct the ground state phase diagrams, we relax systems from these initial states under the LLG equation, until the system has settled into a local (or global) minima in the energy landscape. States are considered to be in equilibrium, and simulations are stopped, when the value of $|\partial \mathbf{m} / \partial t|$ is less than a tolerance of 0.01 degrees per nanosecond, at which point the magnetization is no longer changing. We use a damping factor value of $\alpha=1$ in order to achieve convergence to the final states quickly, by suppressing the precessional dynamics, which does not affect the final state. Once the dynamics have subsided according to the above criterion, we compute the total energy of these relaxed states. We identify the lowest energy state that we have found (from the set of simulations starting from different initial configurations) as the ground state for the given geometry and applied field value, which allows us to construct $d-B$ phase diagrams of the ground states. We consider the higher energy relaxed states to be metastable.

To perform the simulations, we use the finite-element micromagnetic simulator Finmag, developed at the University of Southampton. This uses the DOLFIN component of the finite-element solver FEniCS, ${ }^{39}$ and integrators from the CVODE component of the SUNDIALS library $\stackrel{40}{40}$ 
(a) Squares
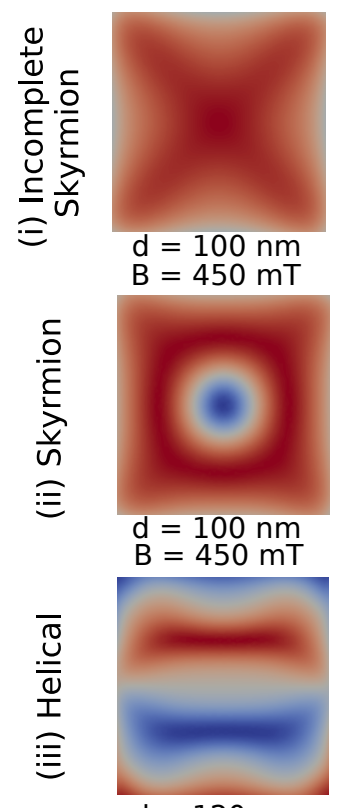

$\mathrm{d}=120 \mathrm{~nm}$

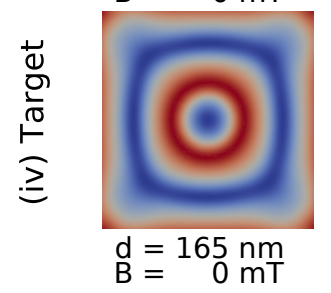

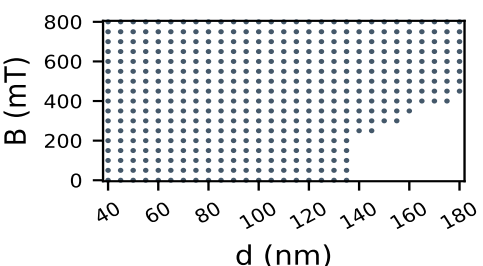
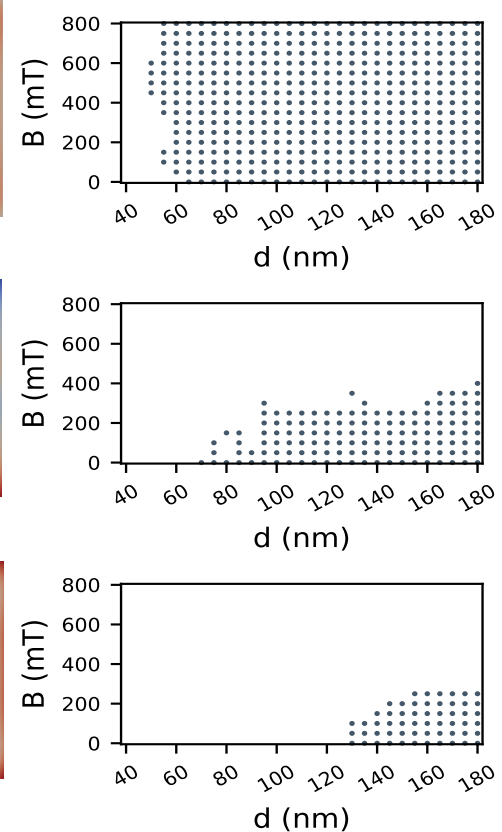

(b) Triangles

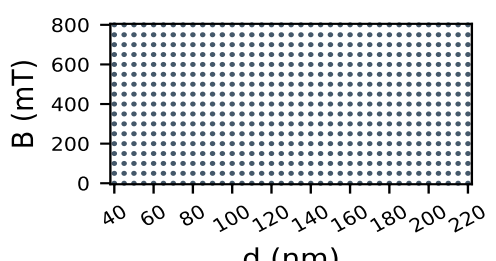

d $(\mathrm{nm})$
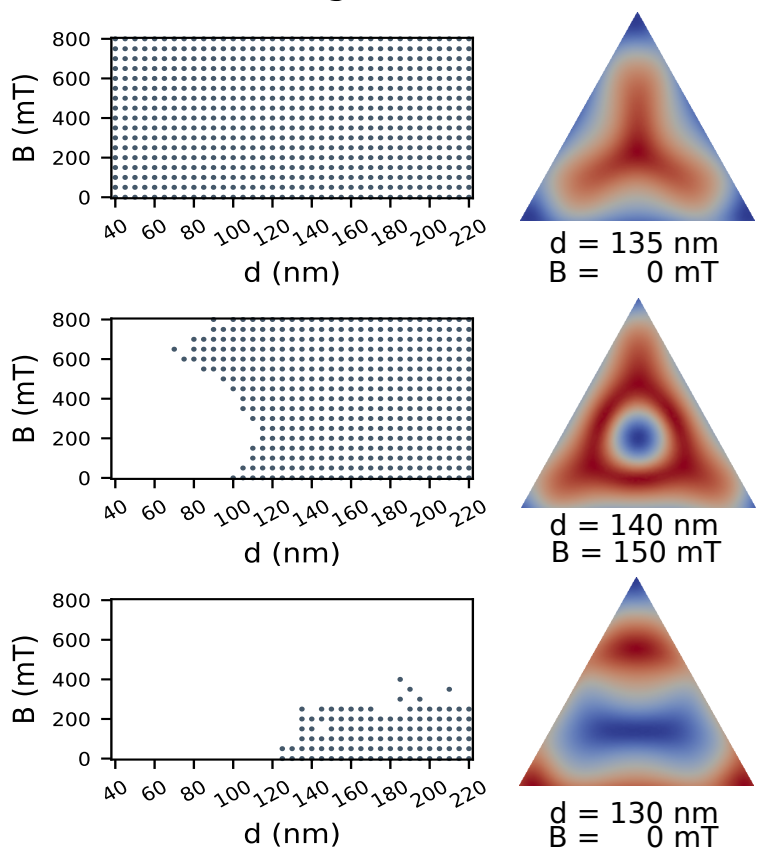

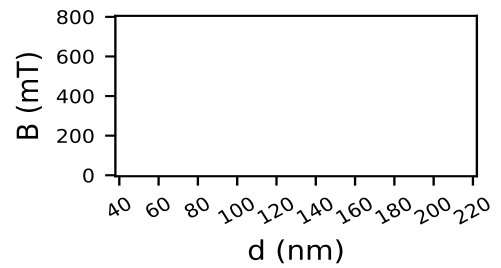

FIG. 2. Equilibrium regions for states found in different geometries. In the first and last columns we show the z-component of the magnetization for examples of states (i) to (iv) in the square and triangle systems. The $d$ - $B$ graphs show dots when a metastable state of that type was found for that size and applied field. Incomplete skyrmion states (top row), are not stable for large square systems, with a field lower than around $400 \mathrm{mT}$. However, this is not seen in triangles. We note that in contrast to squares, we do not see target states as metastable in any region of phase space studied in triangles.

\section{RESULTS}

\section{A. Equilibrium States}

A wide variety of equilibrium states (formed of both the ground and metastable states) are obtained from the simulations in the systems, and in Fig. 2. we show the regions in $d-B$ phase space where each state can form as an equilibrium state. The equilibrium states can be broadly classified into several groups.

1. Incomplete Skyrmions - These states are named 27 as such due to the presence of a quasi-uniform magnetization across the system. Due to the DMI, at the boundaries we see twisting of the magnetization. (Fig. 2 (i))

2. Isolated Skyrmions These states, normally axially symmetric in disks, are distorted by the boundary of the confined geometry in both triangular and square systems. (Fig. 2 (ii))
3. Helical States A large variety of rotational spin textures form metastable states in the studied systems. (Fig. 2 (iii))
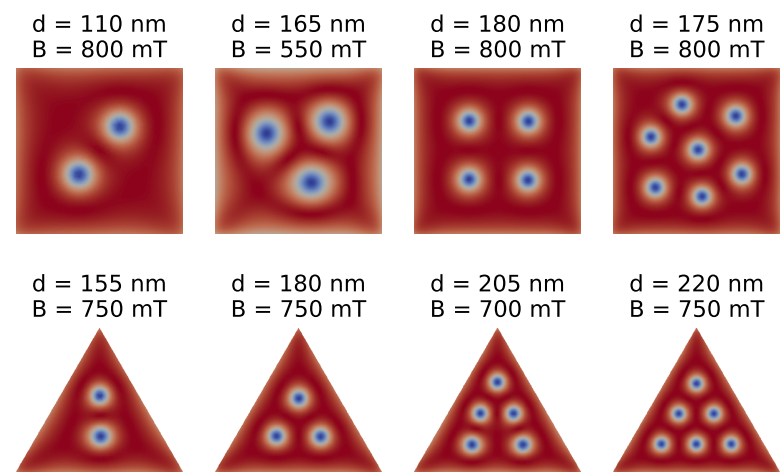

FIG. 3. Examples of the z-component of the magnetization for high energy states containing multiple skyrmions. For square systems, we saw states containing up to 10 skyrmions, and in triangles, a maximum of 6 skyrmions were observed. 


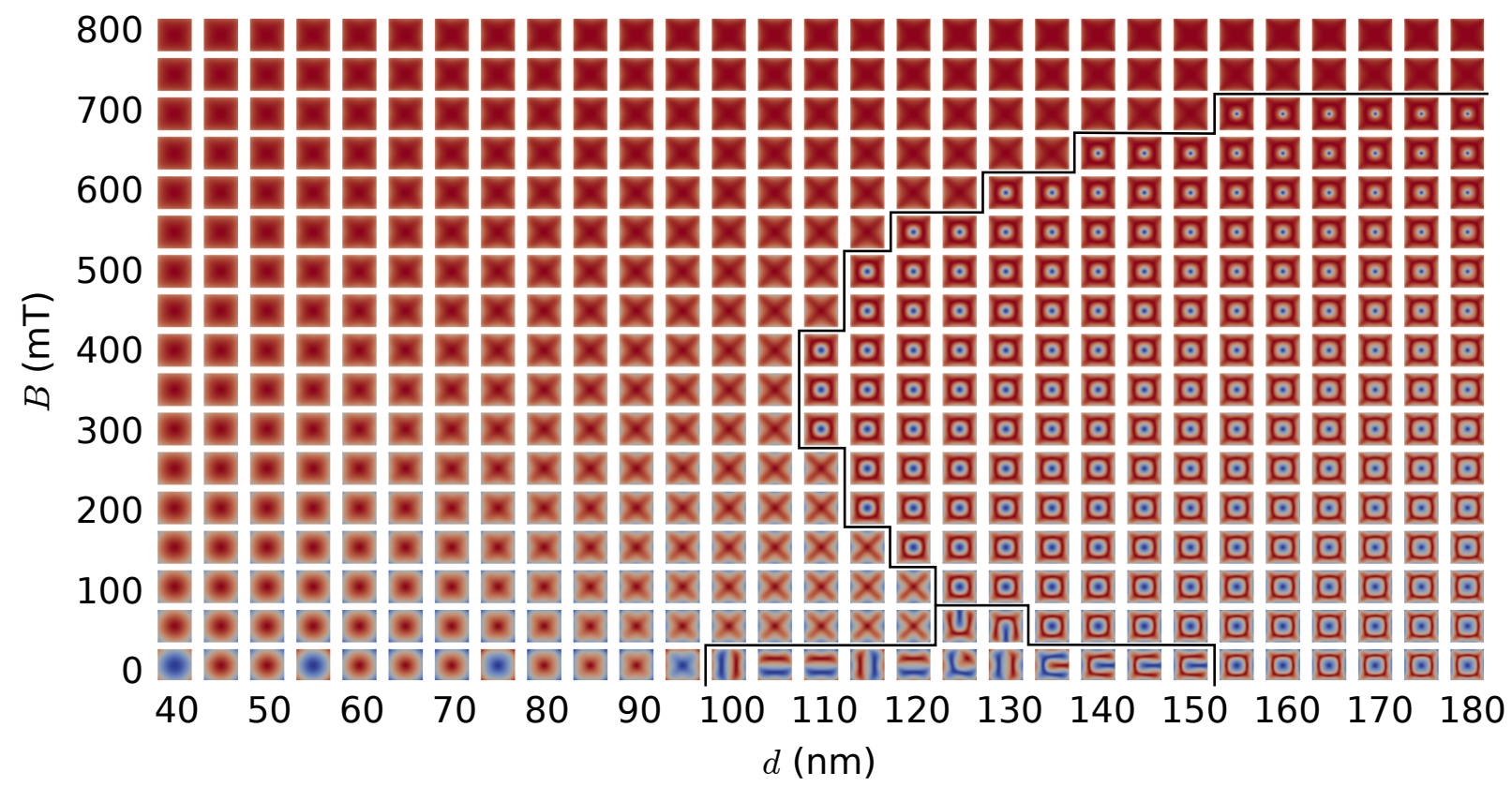

FIG. 4. In this $d-B$ ground state phase diagram for the square geometry, we show the z-component of the magnetization for the lowest energy state found for each sample size and applied field value. We see three regions of interest. (a) The incomplete skyrmion state forms the bulk of the phase diagram. (b) For a narrow region and for low applied field values, we see that helical states form the ground state. (c) Isolated skyrmions form the ground state for large sample sizes. As the field is increased, we can see the skyrmion shrinks such that more of the system aligns with the applied field, and for high field values the skyrmion no longer forms the ground state.

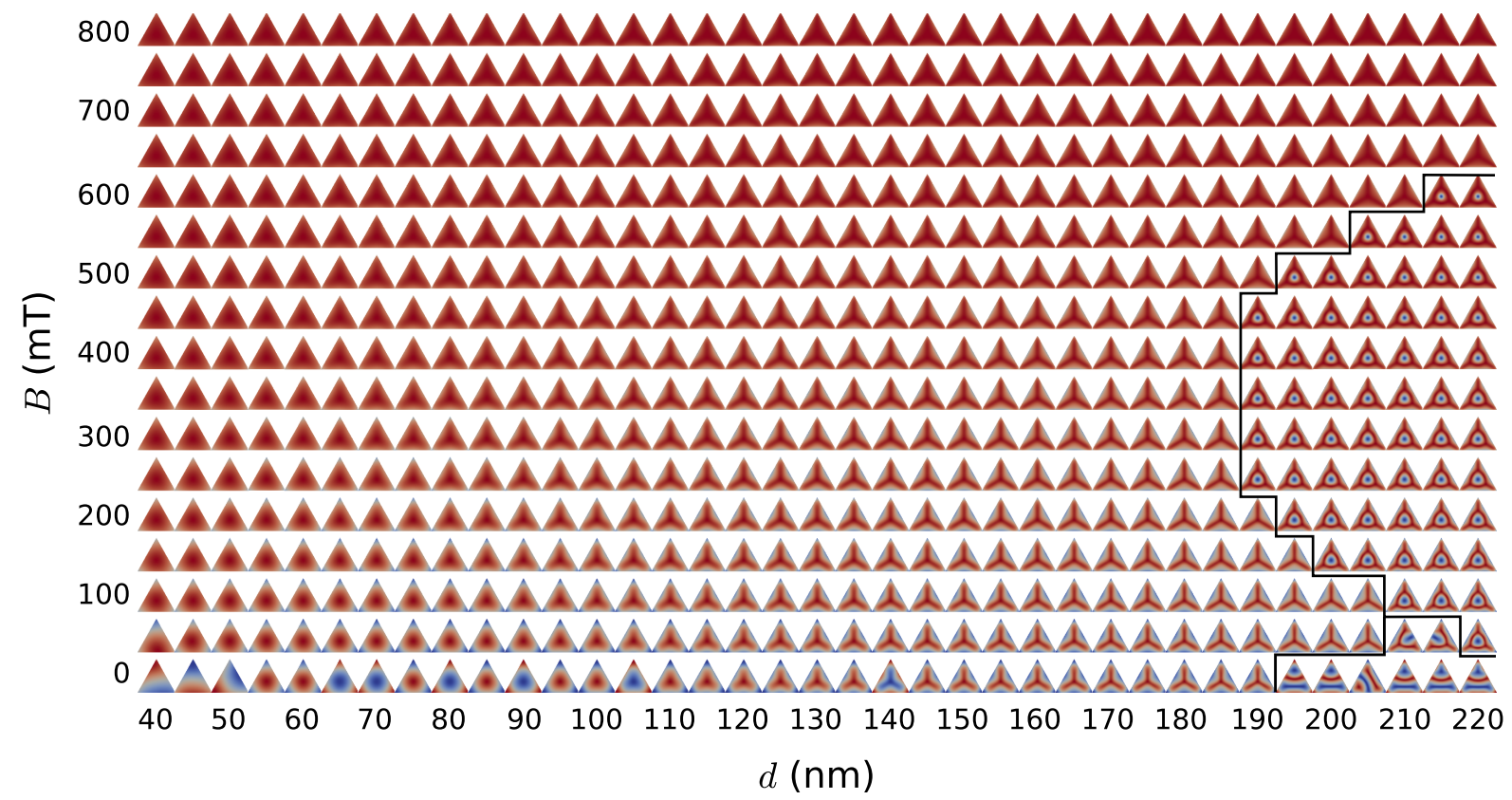

FIG. 5. Here, we show the $d-B$ ground state phase diagram for the triangle geometry, with the z-component of the magnetisation shown for each state obtained. As in Fig. 4 we see three regions of interest. (a) The incomplete skyrmion state forms the bulk of the phase diagram. (b) For very large triangles, again at low applied field values, we see that helical states form the ground state. (c) Skyrmions form the ground state for large sample sizes, but we do not observe these without an applied field. 
(a)

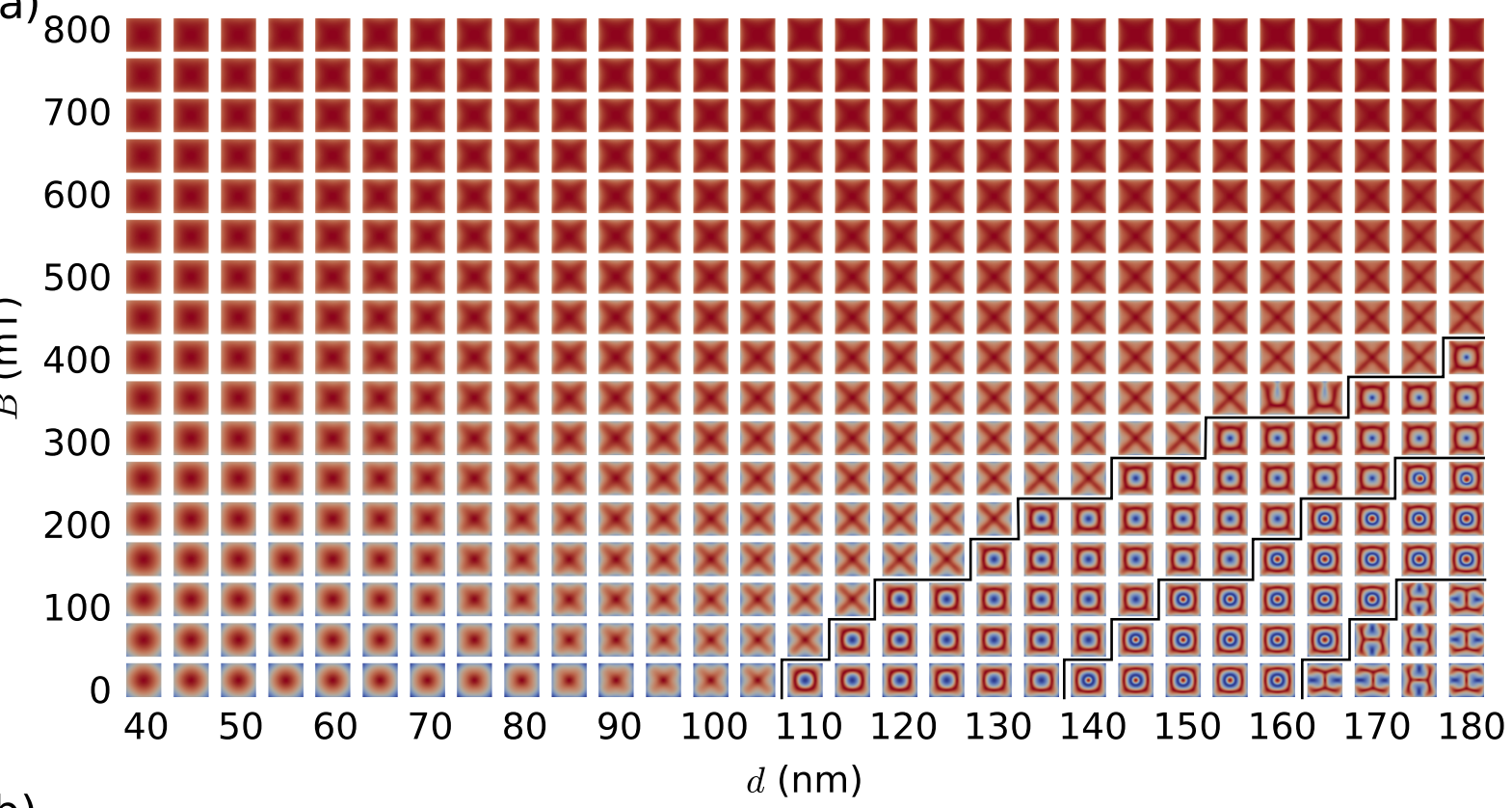

(b)

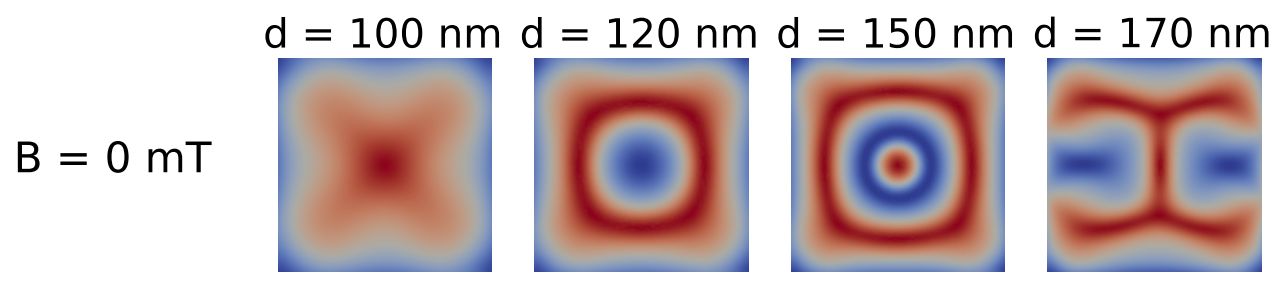

FIG. 6. Obtained states from relaxing square systems from uniform magnetization. In (a), we see four regions of different type of states - from left to right (i) incomplete skyrmions (ii) isolated skyrmions (iii) overcomplete skyrmions, and (iv) helical type states. We predict that hese configurations can be achieved in an experimental study where first a high saturation field is applied in the out-of-plane direction, and then the field is reduced to the value shown on the $y$-axis. In (b), we show the final state obtained for each of these configurations with no applied field.

4. Target States Target states can be considered as an isolated skyrmion, with an additional radial halfhelical rotation. (Fig. 2 (iv))

5. Skyrmion Clusters Multiple clusters of skyrmions form metastable states in the geometries when strong fields are applied to the system, resulting in a smaller skyrmion radius. We find these as highenergy metastable states for larger system sizes and for high applied fields. (Fig. 3)

\section{B. Ground States}

The ground state phase diagrams (Figs. 4 and 5 show the lowest energy states identified for each geometry size for a given applied field. For the square systems we see a large region where isolated skyrmions form the lowest energy state for sample sizes as low as $110 \mathrm{~nm}$ with an applied field of $350 \mathrm{mT}$. For larger sizes, the range of applied fields where skyrmions form the ground state increases, and at $155 \mathrm{~nm}$, we compute that the skyrmion is the ground state with no applied field. For all sample sizes studied, we see that applied fields of above $700 \mathrm{mT}$ result in nearly uniform magnetization. With no applied field, from $100 \mathrm{~nm}$ to $150 \mathrm{~nm}$ we see several types of helical states form the ground state. These results are qualitatively similar to those seen in disk systems, though in disks, skyrmions formed the ground state with no applied field for smaller systems than in squares, with observation at disks of diameter greater than $135 \mathrm{~nm} \cdot 27$

In Fig. 5 , the ground state phase diagram for the triangular systems is shown. In contrast to the square systems, we do not identify skyrmions as the ground state when no applied field is applied for any sized sample we investigated, which shows a strong indication that the shape of the boundary of the system plays a crucial role in the energetics of magnetic skyrmions in confined geometries. Skyrmion states do form the ground state for systems of side length $d>185 \mathrm{~nm}$ when an applied field between 50 and $600 \mathrm{mT}$ is present. For systems of side length $d>190 \mathrm{~nm}$, we see a number of helical states form the ground state with no field. We note that between 40 and $50 \mathrm{~nm}$, we see quasi-helical type states, though the lengths in these systems are below the helical length of 
(a)

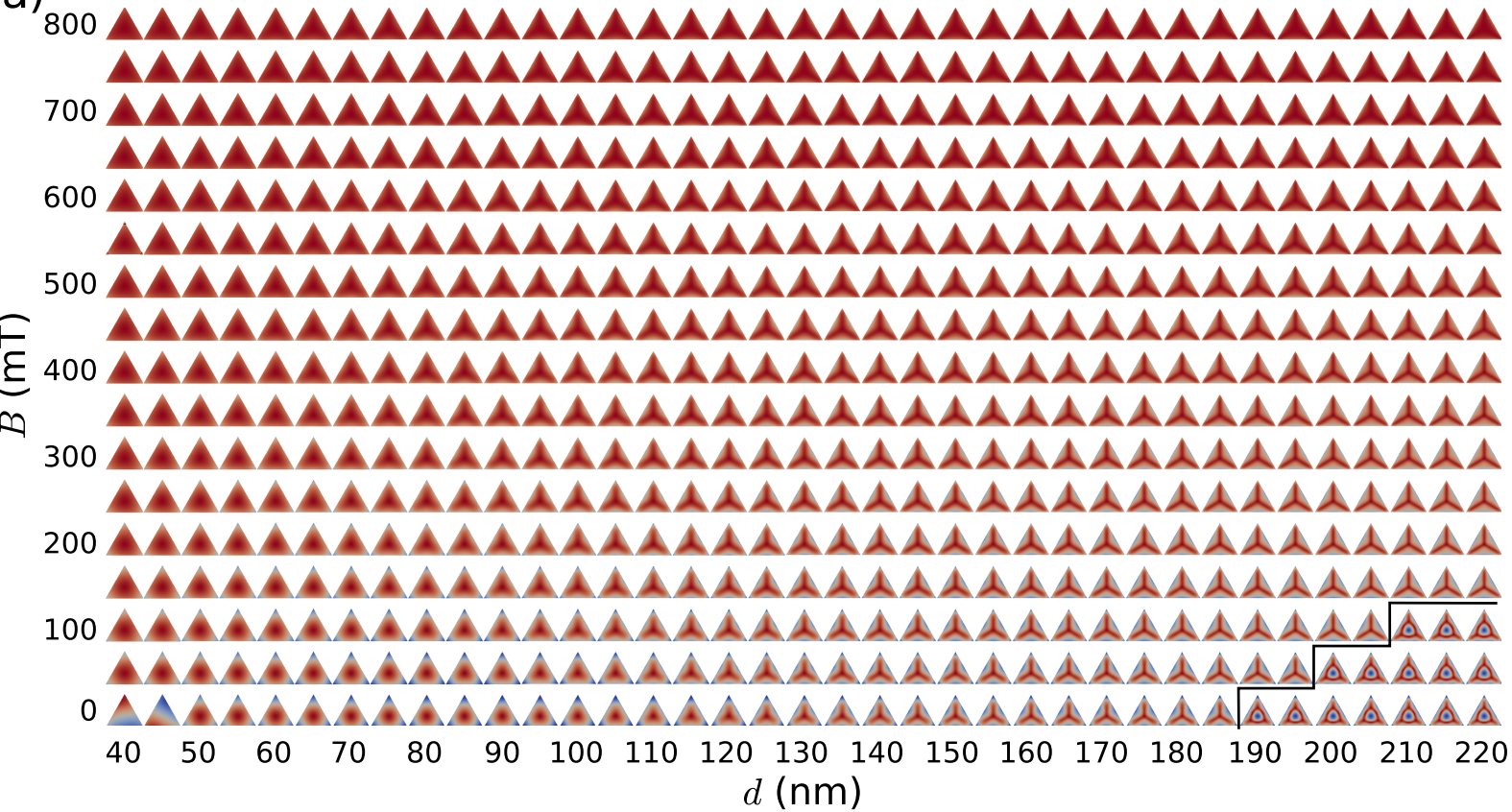

(b)

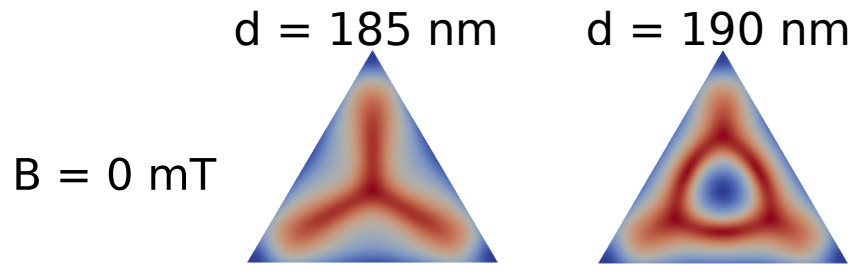

FIG. 7. Here we show the z-component of the magnetization for the states obtained by relaxing triangular systems from uniform magnetization. In (a), we see two regions of different type of states - from left to right (i) incomplete skyrmions (ii) isolated skyrmions. In (b), we show the final state obtained for each of these configurations with no applied field.

FeGe.

The incomplete skyrmion states identified in the triangular geometry vary significantly depending on the size of the systems. Notably, tilting of the magnetization at the boundary of the sample due to the DM interaction causes the magnetization to point most strongly along the axis of the applied applied field, with the strongest alignment along the axes of symmetry in both the square and triangular states, which can be seen in the incomplete skyrmion images shown in Fig. 2(i).

Previously, studies have calculated that in the absence of the demagnetizing field, a requirement for Skyrmions to form the ground state in a system is that either an applied field or a uniaxial anisotropy must be present, in order to stabilize the structure ${ }^{3 / 41}$ Here, however, we show that in squares as in disks, when the system is a confined geometry, we do not require a uniaxial anisotropy, even with no applied field present. We also show that in triangles, we do not see skyrmions form the ground state at zero field. If a uniaxial anisotropy was applied in the out of plane direction, we simply expect that the phase diagram would shift downwards, because the application of an anisotropy in this direction would in effect shift the total internal energy across the whole sample, and in the same way as applying a stronger field.

The Exchange stiffness parameter $A$ and the Dzyaloshiinski-Moriya Interaction constant D are the controlling parameters which affect the radius of the skyrmion 25 . We therefore conclude that varying these parameters would change the skyrmion size, and hence the required nanostructure size necessary to host a skyrmion.

\section{Proposed experimental study}

Of additional interest are states obtained from relaxing the systems from the uniform state. Experimentally, these states could be realised by initially applying a very strong applied field, to ensure that the magnetization of a sample is saturated, and then rapidly reducing the applied magnetic field. The states obtained from doing this in the square sample are shown in Fig. 6. We see four distinct bands of states, with incomplete skyrmions forming the bulk of the phase diagram. Skyrmion states are obtained in a narrow band, between 110 and $135 \mathrm{~nm}$, with no applied field, and at larger sizes of system up to 
$180 \mathrm{~nm}$ with an applied field of $400 \mathrm{mT}$. For system sizes, from 140 to $160 \mathrm{~nm}$ with no applied field, we see target states, and at $165 \mathrm{~nm}$ and above with no applied field, we identify helical states.

The corresponding uniform applied field results for triangular systems is shown in Fig. 7. Here, we see similar results; in the bulk of the phase diagram we see incomplete skyrmion states. For large systems of between 190 and $220 \mathrm{~nm}$, when the field is reduced to a value between 0 and $150 \mathrm{mT}$, we see a small band of skyrmion states.

\section{SUMMARY}

We show in this paper through micromagnetic simulations that in $10 \mathrm{~nm}$ thick confined geometries of FeGe, skyrmions can form the lowest energy state. When there is no applied field, there exists a lower bound of side length $d$ between 150 and $155 \mathrm{~nm}$, below which skyrmions do not form in square systems, and between $100 \mathrm{~nm}$ and $150 \mathrm{~nm}$, a variety of helical type states form the ground state. In triangular systems, we see the incomplete skyrmion state forms the ground state in most of the phase space studied, and in large systems skyrmions form the ground state between fields of 50 and $600 \mathrm{mT}$.

We show over the same range of sizes and fields studied, a wide variety of states are in equilibrium, and we show where these states can be obtained. We present the states obtained from relaxing uniformly magnetised states from the saturated state, in both the square and triangular systems in order to motivate experimental work on FeGe confined geometries, and predict that skyrmion states should be experimentally accessible in both square and triangular systems. We also predict that in large square systems, target states should be accessible using the same procedure.

\section{DATA ACCESS STATEMENT}

All data supporting this study are openly available from the Zenodo repository at https://doi.org/10.5281/zenodo.1066791.

\section{ACKNOWLEDGEMENTS}

This work was financially supported by EPSRC Doctoral Training Centre grant EP/L015382/1, EPSRC Doctoral Training Centre Grant EP/G03690X/1, OpenDreamKit Horizon 2020 European Research Infrastructures project (676541), and the EPSRC Programme grant on Skyrmionics (EP/N032128/1). D.C-O acknowledgees the financial support from CONICYT Chilean scholarship programme Becas Chile (72130061). We acknowledge the use of the University of Southampton
IRIDIS High Performance Computing Facility. T.K. acknowledges financial support from the Gordon and Betty Moore Foundation. W.W. acknowledges the financial support of the National Natural Science Foundation of China (Grant No. 11604169).

\section{REFERENCES}

${ }^{1}$ A. Bogdanov and A. Hubert, Journal of Magnetism and Magnetic Materials 138, 255 (1994).

${ }^{2} \mathrm{~A}$. Bogdanov and A. Hubert, Journal of Magnetism and Magnetic Materials 195, 182 (1999).

${ }^{3}$ U. K. Rößler, A. N. Bogdanov, and C. Pfleiderer, Nature 442, 797 (2006)

${ }^{4}$ S. Muhlbauer, B. Binz, F. Jonietz, C. Pfleiderer, A. Rosch, A. Neubauer, R. Georgii, and P. Boni, Science 323, 915 (2009)

${ }^{5}$ M. Lee, W. Kang, Y. Onose, Y. Tokura, and N. P. Ong, Physical Review Letters 102, 1 (2009)

${ }^{6}$ C. Pfleiderer, A. Neubauer, S. Mühlbauer, F. Jonietz, M. Janoschek, S. Legl, R. Ritz, W. Münzer, C. Franz, P. G. Niklowitz, T. Keller, R. Georgii, P. Böni, B. Binz, A. Rosch, U. K. Rößler, and a. N. Bogdanov, Journal of Physics: Condensed Matter 21, 279801 (2009)

'A. Neubauer, C. Pfleiderer, B. Binz, A. Rosch, R. Ritz, P. G. Niklowitz, and P. Boni, Physical Review Letters 102, 1 (2009) ${ }^{8}$ F. Jonietz, S. Muhlbauer, C. Pfleiderer, A. Neubauer, W. Munzer, A. Bauer, T. Adams, R. Georgii, P. Boni, R. A. Duine, K. Everschor, M. Garst, and A. Rosch, Science 330, 1648 (2010)

${ }^{9}$ T. Adams, A. Chacon, M. Wagner, A. Bauer, G. Brandl, B. Pedersen, H. Berger, P. Lemmens, and C. Pfleiderer, Physical Review Letters 108, 237204 (2012)

${ }^{10}$ S. L. Zhang, A. Bauer, D. M. Burn, P. Milde, E. Neuber, L. M. Eng, H. Berger, C. Pfleiderer, G. van der Laan, and T. Hesjedal, Nano Letters 16, 3285 (2016)

${ }^{11}$ A. Crépieux and C. Lacroix, Journal of Magnetism and Magnetic Materials 182, 341 (1998).

${ }^{12}$ S. Heinze, K. von Bergmann, M. Menzel, J. Brede, A. Kubetzka, R. Wiesendanger, G. Bihlmayer, and S. Blügel, Nature Physics 7, 713 (2011)

${ }^{13}$ C. Moreau-Luchaire, C. Moutas, N. Reyren, J. Sampaio, C. A. F. Vaz, N. Van Horne, K. Bouzehouane, K. Garcia, C. Deranlot, P. Warnicke, P. Wohlhüter, J.-M. George, M. Weigand, J. Raabe, V. Cros, and A. Fert, Nature Nanotechnology 11, 1 (2016)

${ }^{14}$ H. J. Richter, Journal of Physics D: Applied Physics 40, R149 (2007)

${ }^{15}$ A. Fert, V. Cros, and J. Sampaio, Nature Nanotechnology 8, $152(2013)$

${ }^{16}$ P. F. Bessarab, V. M. Uzdin, and H. Jónsson, Computer Physics Communications 196, 335 (2015)

${ }^{17}$ D. Cortés-Ortuño, W. Wang, M. Beg, R. A. Pepper, M.-A. Bisotti, R. Carey, M. Vousden, T. Kluyver, O. Hovorka, and H. Fangohr, Scientific Reports 7, 4060 (2017)

${ }^{18}$ N. Romming, C. Hanneken, M. Menzel, J. E. Bickel, B. Wolter, K. von Bergmann, A. Kubetzka, and R. Wiesendanger, Science 341, 636 (2013)

${ }^{19}$ X. Yu, N. Kanazawa, W. Zhang, T. Nagai, T. Hara, K. Kimoto, Y. Matsui, Y. Onose, and Y. Tokura, Nature Communications 3, 988 (2012)

${ }^{20}$ X. Zhang, G. P. Zhao, H. Fangohr, J. P. Liu, W. X. Xia, J. Xia, and F. J. Morvan, Scientific Reports 5, 7643 (2015)

${ }^{21}$ S. Zhang, J. Wang, Q. Zheng, Q. Zhu, X. Liu, S. Chen, C. Jin, Q. Liu, C. Jia, and D. Xue, New Journal of Physics 17, 023061 (2015)

${ }^{22}$ C. P. Chui and Y. Zhou, AIP Advances 5 (2015), $10.1063 / 1.4930904$

${ }^{25}$ S. Woo, K. Litzius, B. Krüger, M.-Y. Im, L. Caretta, K. Richter, M. Mann, A. Krone, R. M. Reeve, M. Weigand, P. Agrawal, 
I. Lemesh, M.-A. Mawass, P. Fischer, M. Kläui, and G. S. D. Beach, Nature Materials 15, 501 (2016)

${ }^{24}$ S. Krause and R. Wiesendanger, Nature Materials 15, 493 (2016)

${ }^{25}$ S. Rohart and A. Thiaville, Physical Review B 88, $184422(2013)$

${ }^{26}$ V. M. Uzdin, M. N. Potkina, I. S. Lobanov, P. F. Bessarab, and H. Jónsson, Physica B: Condensed Matter (2017), 10.1016/j.physb.2017.09.040

${ }^{2}$ M. Beg, R. Carey, W. Wang, D. Cortés-Ortuño, M. Vousden, M.-A. Bisotti, M. Albert, D. Chernyshenko, O. Hovorka, R. L. Stamps, and H. Fangohr, Scientific Reports 5, 17137 (2015).

${ }^{28}$ C. P. Chui, F. Ma, and Y. Zhou, AIP Advances 5 (2015), $10.1063 / 1.4919320$

${ }^{29}$ X. Zhao, C. Jin, C. Wang, H. Du, J. Zang, M. Tian, R. Che, and Y. Zhang, Proceedings of the National Academy of Sciences 113, 4918 (2016)

${ }^{{ }^{0}}$ F. Zheng, H. Li, S. Wang, D. Song, C. Jin, W. Wei, A. Kovács, J. Zang, M. Tian, Y. Zhang, H. Du, and R. E. Dunin-Borkowski, Physical Review Letters 119, 197205 (2017)

${ }^{31}$ R. Carey, M. Beg, M. Albert, M.-A. Bisotti, D. Cortés-Ortuño, M. Vousden, W. Wang, O. Hovorka, and H. Fangohr, Applied Physics Letters 109, 122401 (2016)

${ }^{52}$ F. N. Rybakov, A. B. Borisov, S. Blügel, and N. S. Kiselev, New Journal of Physics 18, 045002 (2016)
${ }^{33}$ M. Vousden, M. Albert, M. Beg, M.-A. Bisotti, R. Carey, D. Chernyshenko, D. Cortés-Ortuño, W. Wang, O. Hovorka, C. H. Marrows, and H. Fangohr, Applied Physics Letters 108, $132406(2016)$

${ }^{34}$ A. O. Leonov, Y. Togawa, T. L. Monchesky, A. N. Bogdanov, J. Kishine, Y. Kousaka, M. Miyagawa, T. Koyama, J. Akimitsu, T. Koyama, K. Harada, S. Mori, D. McGrouther, R. Lamb, M. Krajnak, S. McVitie, R. L. Stamps, and K. Inoue, Physical Review Letters 117, 1 (2016)

${ }^{35}$ S. Schneider, D. Wolf, M. J. Stolt, S. Jin, D. Pohl, B. Rellinghaus, M. Schmidt, B. Büchner, S. T. B. Goennenwein, K. Nielsch, and A. Lubk, (2017), arXiv:1710.08322

${ }^{36}$ D. Fredkin and T. Koehler, IEEE Transactions on Magnetics 26, $415(1990)$

"3/B. Lebech, J. Bernhard, and T. Freltoft, Journal of Physics: Condensed Matter 1, 6105 (1989).

${ }^{38}$ J. C. Gallagher, K. Y. Meng, J. T. Brangham, H. L. Wang, B. D. Esser, D. W. McComb, and F. Y. Yang, Physical Review Letters 118, 027201 (2017)

${ }^{39}$ A. Logg and G. N. Wells, ACM Transactions on Mathematical Software (TOMS) 37, 2 (2010)

${ }^{40}$ A. C. Hindmarsh, P. N. Brown, K. E. Grant, S. L. Lee, R. Serban, D. E. Shumaker, and C. S. Woodward, ACM Transactions on Mathematical Software 31, 363 (2005)

${ }^{41}$ K. Y. Guslienko, IEEE Magnetics Letters 6 (2015) 